\title{
Planck LFI: Comparison between Galaxy Straylight Contamination and other systematic effects
}

\author{
C. Burigana ${ }^{1}$, D. Maino ${ }^{2}$, K. M. Górski ${ }^{3,4}$, N. Mandolesi $^{1}$, M. Bersanelli ${ }^{5}$, F. Villa $^{1}$, L. Valenziano $^{1}$, \\ B. D. Wandelt ${ }^{6}$, M. Maltoni ${ }^{7}$, and E. Hivon ${ }^{8,9}$ \\ 1 Istituto TeSRE, Consiglio Nazionale delle Ricerche, via Gobetti 101, 40129 Bologna, Italy \\ 2 Oss. Astr. Trieste, via G.B. Tiepolo 11, 34131 Trieste, Italy \\ 3 ESO, European Southern Observatory, Karl-Schwarzschild Str. 2, 85748 Garching, Germany \\ 4 Warsaw University Observatory, Warsaw, Poland \\ 5 Dipartimento di Fisica, Università di Milano, and IFC/CNR, via Celoria 16, 20133 Milano, Italy \\ 6 Department of Physics, Princeton University, Princeton, NJ 08544, USA \\ 7 Instituto de Física Corpuscular - CSIC/UVEG, Edificio Institutos de Paterna, Apt. 22085, \\ 46071 Valencia, Spain \\ 8 Observational Cosmology, California Institute of Technology, MS 59-33, CA 91125 Pasadena, USA \\ 9 IPAC, California Institute of Technology, MS 100-22, CA 91125 Pasadena, USA
}

Received 2 October 2000 / Accepted 14 March 2001

\begin{abstract}
The straylight contamination due to the Galactic emission (GSC, Galaxy Straylight Contamination) entering at large angles from the antenna centre direction may be one of the most critical sources of systematic effects in observations of the cosmic microwave background (CMB) anisotropies by future satellite missions such as PLANCK and MAP. While future accurate measurements of the real antenna pattern are necessary for a firm evaluation of this effect, on the basis of the currently available optical simulations we are able to produce simulated data useful for the development of data analysis methods and the optimization of the optical design. For the Low Frequency Instrument (LFI), the GSC is expected to be particularly crucial at the lowest frequency channels. We describe here different methods to evaluate the impact of this effect and compare it with other systematics of instrumental and astrophysical origin. The results are presented in terms of simulated data streams and maps, Fourier series decomposition and angular power spectrum. The contributions within a few degrees from the beam centre dominate the GSC near the Galaxy plane. The antenna sidelobes at intermediate and large angles from the beam centre dominate the GSC at medium and high Galactic latitudes. We find a GSC peak at $\sim 15 \mu \mathrm{K}$ and a GSC angular power spectrum above that of the white noise for multipoles $\ell \lesssim 10$, albeit smaller than that of CMB anisotropies by a factor larger than $\sim 10$. At large multipoles, the GSC affects the determination of CMB angular power spectrum significantly less than other kinds of instrumental systematics, such as main beam distortions and $1 / f$ noise. Although the GSC is largest at low Galactic latitudes, the contamination produced by far pattern features at medium and high Galactic latitudes, peaking at $\sim 4 \mu \mathrm{K}$, has to be carefully investigated, because the combination of low amplitude of Galaxy emission in those regions with the extremely good nominal PLANCK sensitivity naturally makes high Galactic latitude areas the targets for unprecedentedly precise estimation of cosmological CMB anisotropy. This paper is based on PLANCK LFI activities.
\end{abstract}

Key words. cosmology: cosmic microwave background - Galaxy: general - space vehicles - telescopes methods: data analysis

\section{Introduction}

After the great success of COBE-DMR (Smoot et al. 1992; Bennett et al. 1996a; Górski et al. 1996) which probed the gravitational instability scenario for structure formation through the detection of CMB anisotropies at scales of

Send offprint requests to: C. Burigana, e-mail: burigana@tesre.bo.cnr.it few degrees, and the recent balloon-borne experiments at high sensitivity and resolution on limited sky regions (De Bernardis et al. 2000; Hanany et al. 2000), supporting a universe model with $\Omega_{\text {tot }} \sim 1$ (Lange et al. 2000; Balbi et al.2000; Jaffe et al.2000), ultimately, future progress of the CMB anisotropy cosmology will be based on two space missions, MAP (Microwave Anisotropy Probe) (see Bennett et al.1996b) by NASA and PLANCK by ESA, 
planned to be launched respectively in the years 2001 and 2007.

In particular, the Low Frequency Instrument (LFI, Mandolesi et al. 1998) and the High Frequency Instrument (HFI, Puget et al.1998) on-board PLANCK will cover together a wide frequency range $(30 \div 900 \mathrm{GHz})$ which should significantly improve the accuracy of the subtraction of foreground contamination from the primordial CMB anisotropy, providing at the same time a gold-mine of cosmological as well as astrophysical information (see, e.g., De Zotti et al. 1999a and references therein).

To fully reach these scientific goals, great attention has to be devoted to properly reducing and/or subtracting all the possible systematic effects. Detailed simulation codes have been developed and are continuously implemented to analyse the impact of several classes of instrumental effects related to the behaviour of the optics, instruments and environment for a wide set of possible scanning strategies (see, e.g., Burigana et al. 1998; Delabrouille 1998; Maino et al. 1999; Mandolesi et al. 2000a). Ultimately this effort leads to the optimization of the mission design and the production of realistic data streams and simulated maps for data analysis tools as well as a realistic definition of PlANCK's scientific performance.

In particular, the behaviour of the PLANCK antenna patterns, both at intermediate and large angles from the directions of beam centres, have to be carefully considered. The requirement for the rejection of unwanted radiation coming from directions far from the optical axis (straylight) is stringent for PLANCK and does not pertain only to the telescope itself, but to the entire optical system, including also solar panels, shielding, thermal stability and focal assembly components. The primary sources of error for the LFI are those due to imperfect off-axis rejection by the optical system of radiation from the Sun, Earth, Moon, planets, Galaxy and the spacecraft itself (de Maagt et al. 1998). The variations of the spurious straylight signal during the mission introduce contaminations in the anisotropy measurements.

The antenna response features at large angular scales from the beam centre (far sidelobes) are determined largely by diffraction and scattering from the edges of the mirrors and from nearby supporting structures. Therefore they can be reduced by decreasing the illumination at the edge of the primary, i.e. increasing the edge taper, defined as the ratio of the power per unit area incident on the centre of the mirror to that incident on the edge. Of course, the higher the edge taper, the lower the sidelobe level and the straylight contamination. On the other hand, increasing the edge taper has a negative impact on the angular resolution for the fixed size of the primary mirror (see, e.g., Mandolesi et al. 2000a). A trade off between angular resolution and straylight contamination has to be found.

The main aim of this work is to evaluate the impact of the Galactic emission as a source of straylight for PLANCK LFI. We will then compare it with the effects generated by other kinds of systematics, the main beam distortion introduced by optical aberrations and the $1 / f$ noise related to gain fluctuations in LFI radiometers, and with the astrophysical contamination from the Galaxy and the extragalactic sources in the main beam. At LFI frequencies, the Galaxy straylight contamination (GSC) is expected to be particularly crucial at the lowest frequencies, due to the increase of synchrotron absolute emission and anisotropies with the wavelength. For simplicity, we limit our analysis to the case of the $30 \mathrm{GHz}$ channel, but the methods presented here can be extended to higher frequencies.

In Sect. 2 we briefly describe the basic recipes of our simulation code, discussing the geometrical aspects relevant for the full sky convolution, the format of the optical simulation output performed by the ESA (de Maagt et al. 1998) that are adopted in the present work, the conversion from data streams to maps and the computation of the Fourier modes and of the angular power spectra, two different estimators of the GSC impact. We estimated the expected GSC on the basis of the antenna integrated response from angular regions at different angles from the beam centre, and on the level of Galaxy emission. In Sect. 3 we focus on the integration accuracy of our computations and test the consistency of the code by assuming simplified input maps and antenna patterns. The main results concerning the evaluation of the GSC are presented in Sect. 4. In Sect. 5 they are compared with the effects introduced by other kinds of instrumental effects and several sources of astrophysical contamination. Finally, we draw our main conclusions in Sect. 6 .

\section{Simulation of PLANCK observations}

The selected orbit for PLANCK is a Lissajous orbit around the Lagrangian point L2 of the Sun-Earth system (see, e.g., Mandolesi et al.1998). The spacecraft spins at 1 r.p.m. and the field of view of the two instruments is $5^{\circ}$ around the telescope optical axis at a given angle $\alpha$ from the spin-axis direction, given by a unit vector, $s$, chosen outward of the Sun's direction. In this work we consider values of $\alpha \sim 80^{\circ}-90^{\circ}{ }^{1}$. The spin axis will be kept parallel to the Sun-spacecraft direction and repointed by $\simeq 2.5^{\prime}$ every $\simeq 1$ hour. In addition, a precession of the spin-axis with a period, $P$, of $\simeq 6$ months at a given angle $\beta \sim 10^{\circ}$ about an axis, $\boldsymbol{f}$, parallel to the Sun-spacecraft direction (and outward relatively the Sun) and shifted to $\simeq 2.5^{\prime} \mathrm{ev}$ ery $\simeq 1$ hour, may be included in the effective scanning strategy. These kinds of scanning strategies do not modify the angle between the spin axis and the Sun-spacecraft direction, avoiding possible thermal fluctuations induced by modulations of the Sun's illumination, and allow the telescope to achieve nearly full or full sky coverage. Hence PLANCK will trace large circles in the sky. The detailed distribution in the sky of the number of observations per pixel depends on the adopted scanning strategy, the telescope design and the arrangement of the feed array on the telescope focal surface. The scanning strategy and spacecraft geometry have to be carefully addressed in order to

\footnotetext{
1 Most recently it was recommended to choose $\alpha=85^{\circ}$.
} 
minimize systematic effects before and after the data analysis and to ensure that the sky coverage is as complete and spatially smooth as possible.

The code we have implemented for simulating PLANCK observations for a wide set of scanning strategies is described in detail in Burigana et al. $(1997,1998)$ and in Maino et al. (1999). Here we consider simple scanning strategies, namely with the spin axis kept always in the ecliptic plane. Under this assumption, the geometrical input parameters relevant for the scanning strategy are the angle $\alpha$ between the spin axis and the telescope line of sight and the beam location in the telescope field of view. In this study we neglect the small effects introduced on the GSC evaluation by the PLANCK orbit by simply assuming PLANCK located in L2 and consider spin-axis shifts of $2^{\circ}$ every two days and 180 samplings per scan circle. These simplifications allow us to speed up the simulation without significantly affecting our understanding of the main effects introduced by the GSC, because of the decreasing of the Galaxy fluctuation level at small scales and because the effects of pattern features we want to study here occur at $\sim$ degree or larger scales. In fact, a recent study of synchrotron emission (Baccigalupi et al. 2000a) based on recent high resolution surveys at low at medium latitudes indicates a steeper power law of the total intensity angular power spectrum where diffuse emission dominates.

\subsection{Coordinate transformations and input antenna pattern}

For the simple scanning strategies considered here, the vector $s$, which gives the spin axis direction, and a unit vector $\boldsymbol{p}$, parallel to the telescope line of sight $z$, are simple functions of the time and of three scanning strategy parameters: the spinning frequency $f_{\mathrm{s}}$, the angle $\alpha$ and the orientation of the PLANCK telescope line of sight at the beginning of each scan circle that we arbitrarily fix as close as possible to the positive $z$ ecliptic axis. In additon, we set the rotation of $\boldsymbol{p}$ clockwise with respect to the positive direction of $s$.

On the plane tangent to the celestial sphere in the central direction of the field of view, i.e. on the field of view plane of the PLANCK telescope, we choose two coordinates $x$ and $y$, with unit vector $\boldsymbol{u}$ and $\boldsymbol{v}$ respectively, according to the convention that the unit vector $\boldsymbol{u}$ points always toward $s$ and that $x, y, z$ is a standard cartesian frame, referred here as "telescope frame" 2 .

In general, the beam centre will be identified by its unit vector $\boldsymbol{b}$ in the frame $x, y, z$ or equivalently by the coordinates, $x_{0}, y_{0}$, of its projection on the plane $x, y$ or, more usually, by its corresponding standard polar

\footnotetext{
2 We note that the "telescope frame" defined above may be equivalent or not to the "primary mirror frame" depending on the absence or presence of a tilt angle between the primary mirror axis and the central direction of the sky field of view. For example, the current optical configuration forseen for PLANCK exibits a tilt angle of $\simeq-3.75^{\circ}$.
}

coordinates, the colatitude $\theta_{\mathrm{B}}$ and the longitude $\phi_{\mathrm{B}}$. The HFI feedhorns will be located in a circular area at the centre of the focal plane, and LFI feedhorns in a ring around HFI. Therefore the corresponding positions of LFI beams on the sky field of view are significantly off-axis. For a telescope with $\simeq 1.5 \mathrm{~m}$ aperture, the typical $100 \mathrm{GHz}$ LFI beam is located at $\simeq 2.8^{\circ}$ from the optical axis, whereas the $30 \mathrm{GHz}$ beams are at about $\simeq 5^{\circ}$ from it.

The shape of the main beam computed by ESA (de Maagt et al.1998) is provided in a regular equispaced grid on $x, y$ about the beam centre. We can then perform the convolution of the main beam (within a chosen angle from the beam centre) with the sky signal directly in this frame.

According to the standard output of the "GRASP8" code for optical simulations as performed de Maagt et al. (1998), we describe the antenna pattern response, $J$, at large angles from the beam centre by using two standard polar coordinates $\theta_{\mathrm{bf}}$ (between $0^{\circ}$ and $180^{\circ}$ ) and $\phi_{\text {bf }}$ (between $0^{\circ}$ and $360^{\circ}$ ) referred to the "beam frame". This corresponds to the standard cartesian "beam frame" $x_{\mathrm{bf}}, y_{\mathrm{bf}}, z_{\mathrm{bf}}$ which is obtained by the "telescope frame" $x, y, z$ when the unit vector of the axis $z$ is rotated by an angle $\theta_{\mathrm{B}}$ on the plane defined by the unit vector of the axis $z$ and the unit vector $\boldsymbol{b}$ up to reach $\boldsymbol{b}^{3}$. We use here the polar coordinates $\theta_{\mathrm{bf}}, \phi_{\mathrm{bf}}$ for the convolution of the antenna pattern with the sky signal at large angles from the beam centre. For the antenna pattern at "intermediate" (namely up to few degrees from the beam centre) and "far" (namely for the entire solid angle) angular distances from the beam centre, different equispaced grids in $\theta_{\mathrm{bf}}$ are available: more refined for the former, because the response variations are stronger close to the beam centre, and less refined for the latter, where the relevant response variations occur on degree or larger angular scales.

The orientation of these frames as the satellite moves is implemented in the code. For each integration time, we determine the orientations in the sky of the telescope frame and of the beam frame and compute the pattern response in each considered sky direction, thus performing a direct convolution with the sky signal.

\subsection{Optical results}

The telescope design of the Carrier Configuration for PLANCK - the current baseline in which PLANCK and FIRST are lauched together and separate in orbit - is based on the Phase A study. The configuration is a off-axis Gregorian Dragone-Mizuguchi telescope with the main reflector oversized at a 1.5 meter projected aperture to

\footnotetext{
${ }^{3}$ Properly, the standard of "GRASP8" code for optical simulations is to provide the far pattern in terms of a "colatitude" angle $\theta_{\mathrm{bf}}^{\prime}$ (between $-180^{\circ}$ and $180^{\circ}$ ) from the "polar" axis parallel $z_{\mathrm{bf}}$ and an "azimuthal" angle $\phi_{\mathrm{bf}}^{\prime}$ (between $0^{\circ}$ and $180^{\circ}$ ) related to $\theta_{\mathrm{bf}}$ and $\phi_{\mathrm{bf}}$ by: $\phi_{\mathrm{bf}}^{\prime}=\phi_{\mathrm{bf}}$ if $\phi_{\mathrm{bf}} \leq 180^{\circ}$ and $\phi_{\mathrm{bf}}^{\prime}=\phi_{\mathrm{bf}}-180^{\circ}$ if $\phi_{\mathrm{bf}}>180^{\circ} ; \theta_{\mathrm{bf}}^{\prime}=\theta_{\mathrm{bf}}$ if $\phi_{\mathrm{bf}} \leq 180^{\circ}$ and $\theta_{\mathrm{bf}}^{\prime}=-\theta_{\mathrm{bf}}$ if $\phi_{\mathrm{bf}}>180^{\circ}$.
} 
reduce the spillover at the primary mirror. The subreflector axis is tilted at $14^{\circ}$ with respect to the main reflector axis. The off-axis design of the PlANCK telescope (see Fig. 1) introduces particular features in the full sky antenna response. In Fig. 1 a sketch of the symmetry plane of the telescope is given. For simplicity, a feedhorn is located at the centre of the focal plane. Its pattern on the sky is along the direction of the optical axis of the telescope (see bottom panel of Fig. 1). The unwanted ray directions are shown in the upper panel of Fig. 1. The rays labelled with 1 are coming from the sky directly into the feed. The rays labelled with 2 are those scattered into the feed by the subreflector only. These identified regions are the spillover past the subreflector for rays 1 and the spillover past the main reflector for rays 2. Considering the PLANCK Carrier Configuration (de Maagt et al. 1998), the rays $1 \mathrm{~b}$ and $2 \mathrm{~b}$ are blocked by the spacecraft shields, which redirect the rays in an angular region close to the main beam. In Fig. 2 we show a map of the considered full pattern; see the connection beetween the regions marked in Figs. 1 and 2. In Fig. 3 we show also several cuts at constant azimuths $\phi_{\text {bf }}$ from $0^{\circ}$ to $360^{\circ}$ (from the bottom to the top).

We have conservatively considered the worst case (de Maagt et al.1998) for the straylight effect: we use the antenna pattern computed at $30 \mathrm{GHz}$, the channel with the highest spillover and with the highest Galaxy signal. We also included the shields for the Carrier Configuration.

The main feature is the spillover (2a) at about $90^{\circ}$ from the main beam (see also Fig. 2, where the main beam is located at North pole) which shows a response of $\approx-60 \mathrm{~dB}$ with respect to the maximum, i.e. $\approx-10 \mathrm{dBi}$ (the main beam peak level is at $\sim 50 \mathrm{dBi}$ ), extending for few tens of degrees in $\theta_{\mathrm{bf}}$ and in $\phi_{\mathrm{bf}}$ (around $\phi_{\mathrm{bf}}=0^{\circ}$, i.e. always quite close to the direction of the axis $x$ in the "telescope frame"). Another relevant feature is the subreflector spillover (1a), with similar response level and an angular extension close to the main beam $\left(\theta_{\mathrm{bf}} \sim 30^{\circ}\right)$ as we can see in Fig. 1. Other features are also located on the northern semisphere, due to the shields which block rays coming from the southern part of the sphere (see de Maagt et al. 1998 for a more detailed discussion of their connection with the optical configuration).

The pattern has been calculated using the Physical Optics (PO) and its extension, the Physical Theory of Diffraction (PTD). Since pattern responses at levels smaller than about $-60 \mathrm{~dB}$, i.e. $\sim-10 \mathrm{dBi}$, are hard to measure, this is the most accurate method to predict the side lobe response on the antenna. The validity of the simulations at very small levels of the far sidelobes will be tested by measuring the antenna response of a fully representative copy of the PLANCK telescope both in compact range and in outdoor far field test range facilities. Antenna pattern measurements are needed also for the knowledge of the near sidelobes (intermediate pattern, see Sect. 3.1). In fact, mirror distortions and roughness will modify in particular the intermediate pattern shape with respect to the shape calculated with the adopted method that neglects these effects. Moreover, multiple reflections

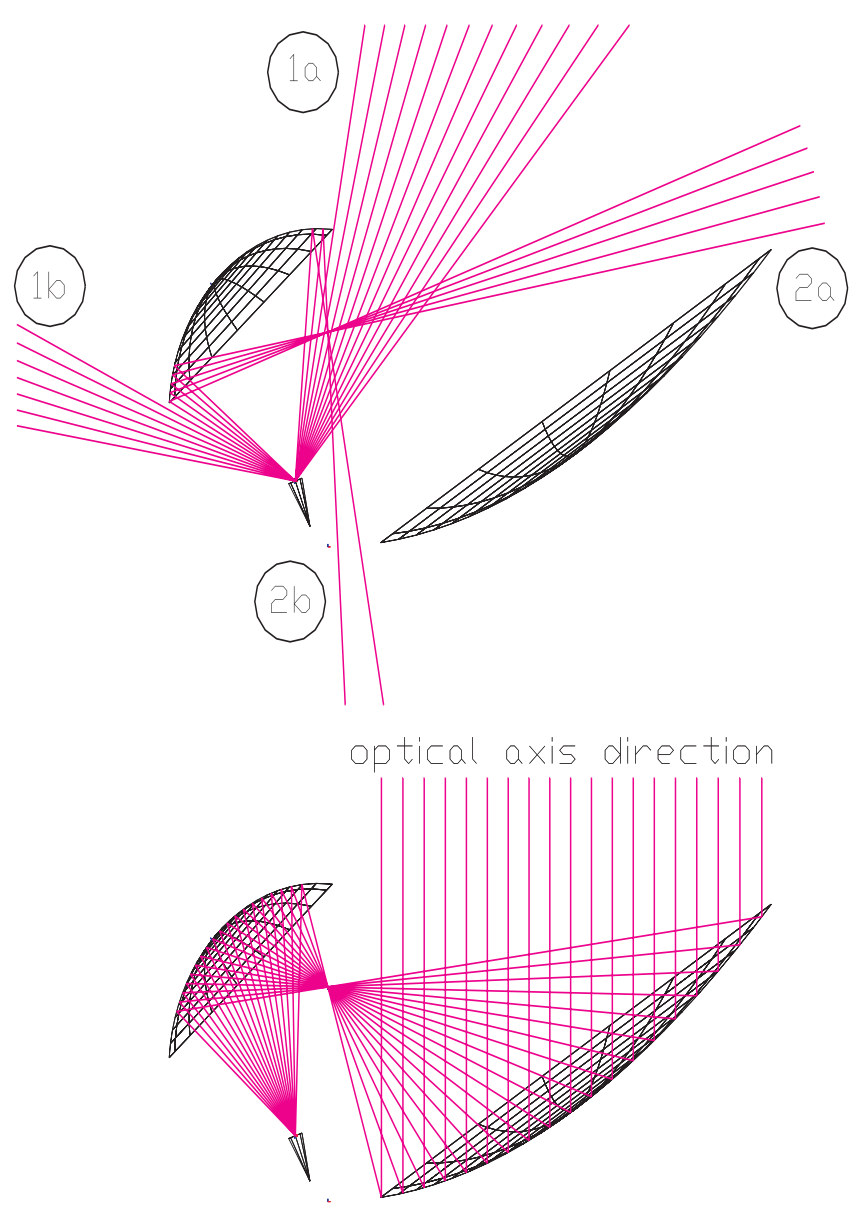

Fig. 1. Sketch of the telescope design in the symmetry plane $\left(\phi_{\mathrm{bf}}^{\prime} \sim 0\right)$. This figure shows the carrier telescope configuration: a gregorian Dragone-Mizuguchi design with the primary mirror enlarged to 1.5 meter to reduce the main spillover (2a). The upper panel shows the rays coming from unwanted directions and focused into the feed: (1a) spillover past the subreflector; (1b) spillover past the subreflector redirect by the shields; (2a) spillover past the main reflector; (2b) spillover past the main reflector and redirect by the shields. The bottom drawing shows the telescope with rays coming from the direction of the optical axis and focused in a feed located at the centre of the focal plane. The spillover blocked by the shield, (1b) and (2b), is redirected close to the main beam as shown on the full-antenna pattern in Fig. 2.

and diffraction on the focal plane unit are not taken into account by the PO/PTD analysis and will modify the intermediate beam shape as well. The realistic prediction of the intermediate pattern is then particularly difficult and the current $\mathrm{PO} / \mathrm{PTD}$ computation provides only a rough estimation of the beam shape in these angular regions.

\subsection{Simple estimates}

Taking the level of Galactic emission and the antenna integrated response from angular regions at different angles from the beam centre, we can provide first order estimates of the expected GSC. 


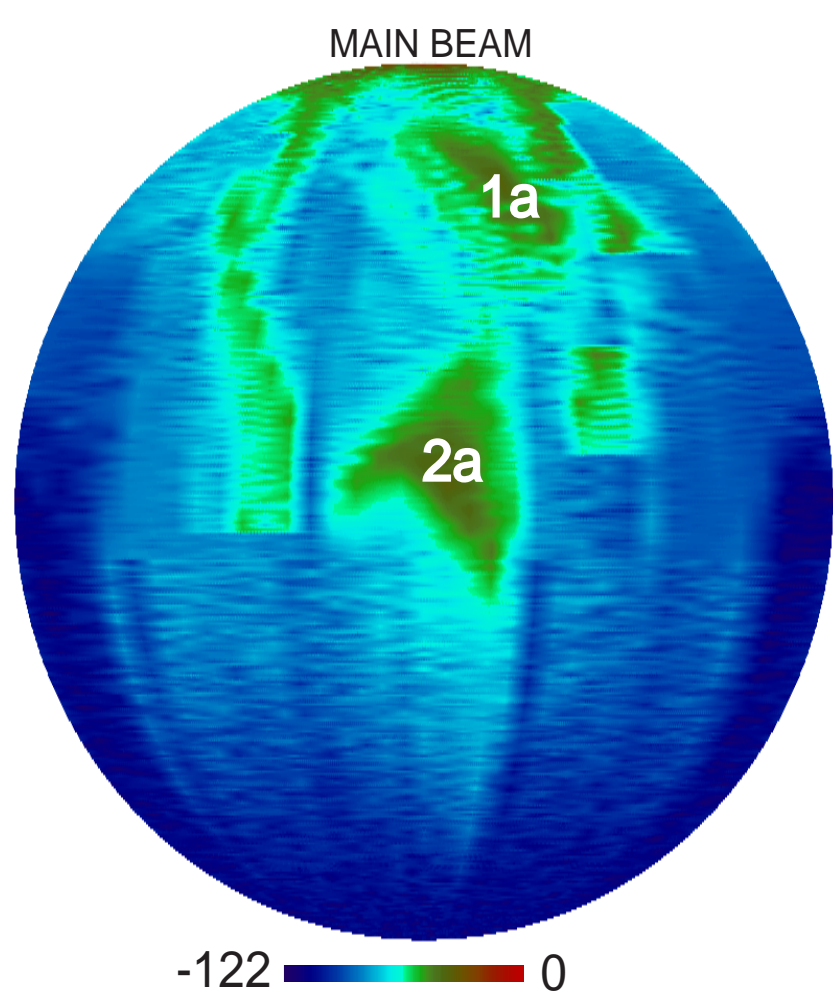

Fig. 2. Full antenna pattern, with response normalized to the maximum, for the carrier configuration. The color table is linear in $\mathrm{dB}$; the true directivity at the maximum is $49.36 \mathrm{dBi}$. Pattern regions related to particular optical structures are identified. See also the text.

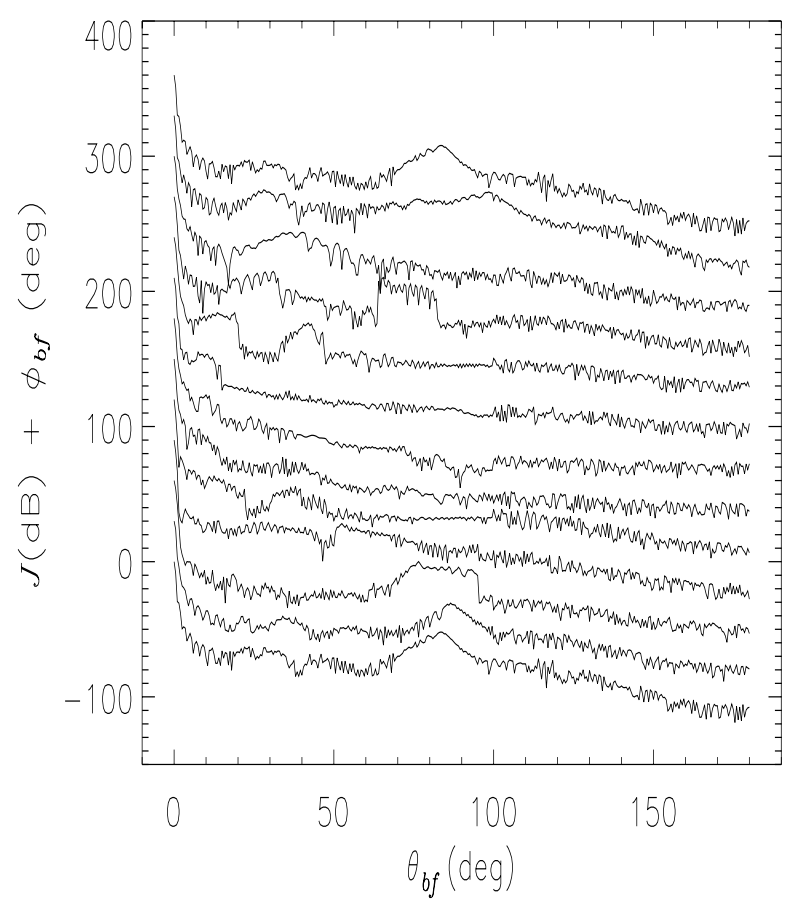

Fig. 3. Cuts of full antenna pattern in $\mathrm{dB}$ for the carrier configuration. The lines refer to the antenna response for $\theta_{\mathrm{bf}}$ between $0^{\circ}$ and $180^{\circ}$ and, from the bottom to the top, with $\phi_{\text {bf }}$ at steps of $30^{\circ}$ from $0^{\circ}$ to $360^{\circ}$ (each cut is vertically shifted for graphic purposes to have the pattern response value at $\theta_{\mathrm{bf}}=0^{\circ}$ equal to the considered value of $\phi_{\mathrm{bf}}$ ).
The region with $\theta_{\mathrm{bf}}$ between $\simeq 1.2^{\circ}$ and $\simeq 2^{\circ}$ contains about $0.5 \%$ of the integrated response; the region between $\simeq 1.2^{\circ}$ and $\simeq 5^{\circ}$ contains about $0.6 \%$ of the integrated response and all the rest of the far pattern $\left(\theta_{\mathrm{bf}} \gtrsim 5^{\circ}\right)$ contains about $1 \%$ of the integrated response. Of course, the remaining main integrated response falls in the "main" beam (up to $\simeq 1.2^{\circ}$ ) (see also Sect. 3.1 for a discussion on the choice of these characteristic angles). In addition, in the main spillover $(2 \mathrm{a})$ enters $\approx 0.1 \div 0.2 \%$ of the integrated response.

The sky signal at $30 \mathrm{GHz}$ is known with a pixel size of about $2.5^{\circ}$ by COBE-DMR. For the present study at $30 \mathrm{GHz}$ the relevant astrophysical source is the Galaxy emission. While the large-scale Galaxy emission at $30 \mathrm{GHz}$ is quite well known, for angular scales smaller then a few degrees we can only resort to extrapolations from existing maps and surveys at lower and higher frequencies. More precise information will be available from the next MAP data and, finally, from PLANCK data themselves. We have here implemented "small" angular extrapolations (see, e.g., Burigana et al. 2000a for further details) for generating Galaxy maps with resolution of about $1.2^{\circ}$, corresponding to Quad-Cube resolution 7.

For simple estimates, we note that in the adopted $30 \mathrm{GHz}$ Galaxy map there are $\sim 13 \square$ with a signal (in terms of antenna temperature $T_{\mathrm{a}}$ ) larger the $2 \mathrm{mK}, \sim 73 \square$ with $T_{\mathrm{a}}>1.5 \mathrm{mK}$ and $\sim 230 \square$ with $T_{\mathrm{a}}>1 \mathrm{mK}$, while the minimum signal is $\sim 0.05 \mathrm{mK}$ and about the $50 \%$ of the sky shows a signal $\sim 0.1 \mathrm{mK}$.

By combining these numbers with the percentages of integrated responses falling within the above different angles from the beam centre, we expect to find a contamination peaking at about $10 \mu \mathrm{K}$ from the pattern regions between $\simeq 1.2^{\circ}$ and $\simeq 5^{\circ}$ and at a few $\mu \mathrm{K}$ from the pattern regions outside $\simeq 5^{\circ}$. In particular, in the main spillover (2a) we expect a signal peaking at $\sim 2 \mu \mathrm{K}$ when it looks at high signal Galactic regions. Similar contributions are expected from the pattern features at few tens of degrees from the beam centre. Of course, smaller contaminations $(\sim 0.5 \mu \mathrm{K})$ are expected when the relevant pattern features look at regions with low Galactic signal.

Numerical calculations, such as those presented in the next sections, are required for more accurate estimates.

\subsection{From data streams to sky maps}

The input map is converted from its original Quad-Cube pixelisation to a equal area, hierarchic HEALPix pixelisation scheme (Górski et al. 1998), adopted in the present work (see also Sect. 3.1 for details about the nominal resolution of this map). The final output of the simulation code relevant here are 2 matrices with a number of rows equal to the considered number of spin-axis positions $n_{\mathrm{s}}$ for one year of mission $\left(n_{\mathrm{s}}=180\right.$ here) and a number of columns equal to the number of considered samplings along one scan circle $\left(n_{\mathrm{p}}=180\right.$ here). In the first matrix, $\mathbf{N}$, we store the pixel numbers corresponding to the main 
beam central directions for the considered $180 \times 180$ integrations; they are stored in HEALPix pixelisation scheme at $n_{\text {side }}=32$ (the number of pixels $n_{\text {pix }}$ in a full sky map is related to $n_{\text {side }}$ by $n_{\text {pix }}=12 n_{\text {side }}^{2}$, Górski et al. 1998). In the second matrix, $\mathbf{G}$, we store the antenna temperatures "observed" by the considered portion of the antenna pattern for the above pointing positions. We neglect here the receiver noise and all the other systematics. These data streams are the first output of our simulations; they give immediately the impact of GSC and are useful to understand how this effect changes during the mission.

From these data streams it is quite simple to obtain simulated observed maps, that can be visualized for example in mollweide projection: we make use of $\mathbf{N}$ and $\mathbf{G}$ to simply coadd the temperatures of those pixels observed several times during the mission. In this way we attribute to each pixel the average of the signals observed when the antenna pattern, due to the scanning strategy, is differently oriented in the sky and thus making Galactic regions with very different signal intensities enter in the intermediate/far sidelobes; of course, by coadding different samples of the same location into pixels the systematic error per pixel is smaller than the systematic error in the most contaminated sample. This is because for different samples of the same location on the sky the sidelobes are pointing towards different regions of the sky, some brighter and some fainter.

\subsection{Power spectra}

We can analyse both data streams and maps in terms of power at different scale-lengths or multipoles (or modes).

In order to analyse separately each scan circle of the simulated data streams we follow the approach suggested by Puget \& Delabrouille (1999) and decompose the time series from the scan circle in Fourier series:

$g(\xi)=\sum_{m=0}^{m=n_{\mathrm{p}} / 2-1} a_{\mathrm{m}} \sqrt{2} \cos \left(m \xi+\xi_{0, \mathrm{~m}}\right)$,

where $g(\xi)$ is the signal at the position identified by an angle $\xi$ (between 0 and $2 \pi$ ) on the considered scan circle. The $2 \times n_{\mathrm{p}}$ coefficients $a_{\mathrm{m}}$ and $\xi_{0, \mathrm{~m}}$ can be easily computed from the time series by solving a linear system. The amplitude, $a_{\mathrm{m}}$, of each mode $\mathrm{m}$, analogous to the multipole $\ell$ of a usual spherical harmonic expansion, has to be compared with that of the wanted signal and of other sources of noise. This approach is particularly interesting for the data analysis during the mission, as PLANCK will continuously scan different circles in the sky.

For analysing the maps of coadded signals we use the standard approach of computing their angular power spectrum. We produce maps in the HEALPix pixelisation scheme (Górski et al. 1998) which takes advantage from the isolatitude of the pixels for a quick generation of a map from the coefficients $a_{\ell m}$ of the spherical harmonic expansion and vice versa (Muciaccia et al. 1997). [We will show the angular power spectra in terms of $\left.\delta T_{\ell}(\nu)=\sqrt{\ell(2 \ell+1) C_{\ell}(\nu) / 4 \pi}\right]$. This is a very significant test for evaluating the GSC impact on PLANCK science, as the estimation of the angular power spectrum of CMB fluctuations from the sky maps is one of the main objectives of the PLANCK mission. It offers also the possibility of directly comparing the GSC with astrophysical contaminations and other sources of instrumental noise. Of course, it produces a "global" estimate of GSC effect, useful in the analysis of the whole sky maps.

\section{Accuracy and tests}

The antenna pattern is theoretically known from optical simulation codes at the desired accuracy and resolution compatible with the available computing time. The adopted grids have a resolution much better than those of currently available sky maps at PLANCK frequencies, although extrapolations both in frequency and in angular resolution of existing maps allow us to produce more refined simulated maps (see, e.g., Burigana et al.1998, 2000a). On the other hand, the knowledge of Galactic emission at high resolution is not particularly relevant here and only small angular extrapolations up to a resolution of about $1^{\circ}$ have been implemented. Given the currently available input map resolution and the adopted simplified scanning strategies, we are able to derive the power of GSC only up to $\ell \sim m \lesssim 80$, a mode/multipole range satisfactory for the study of the smooth features in the intermediate and far pattern response. A very good agreement is found with the high resolution computations based on faster Fourier expansion methods (Wandelt \& Górski 2000) which will be practically necessary for whole sky convolutions at angular resolutions higher than those adopted in this work. On the other hand, at larger multipoles, where a more realistic knowledge of the antenna pattern and the Galaxy emission is necessary for firm predictions (see Sects. 2.2 and 2.3), the GSC effects are dominated by other systematics, as discussed in Sect. 5 .

\subsection{Sky, pattern grids and numerical accuracy}

We are interested in producing signal data streams as they would be observed separately by different angular regions of the antenna pattern in order to understand the effect of the different pattern features as they project in the sky during the PLANCK observations. We have considered here three regions: $0^{\circ} \leq \theta_{\mathrm{bf}} \leq \theta_{\mathrm{bf}, 1}$ (main pattern); $\theta_{\mathrm{bf}, 1} \leq \theta_{\mathrm{bf}} \leq \theta_{\mathrm{bf}, 2}$ (intermediate pattern); $\theta_{\mathrm{bf}, 2} \leq$ $\theta_{\mathrm{bf}} \leq 180^{\circ}$ (far pattern). We have choosen $\theta_{\mathrm{bf}, 1}=1.2^{\circ}$ and $\theta_{\mathrm{bf}, 2}=5^{\circ}$. Of course, the choice of $\theta_{\mathrm{bf}, 1}$ and $\theta_{\mathrm{bf}, 2}$ has to be appropriate to the considered antenna pattern: for a given telescope design it depends mainly on the considered frequency and only weakly on the exact feed location on the focal surface. For the $30 \mathrm{GHz}$ channel, the main beam can be accurately measured in-flight using planet transits (Mandolesi et al. 1998; Burigana et al.2000b) up to response levels of $\approx-30 \mathrm{~dB}(\approx 20 \mathrm{dBi})$ with respect 
to the peak response; $\theta_{\mathrm{bf}} \gtrsim 1.2^{\circ}$ corresponds to antenna responses lower than $-40 \mathrm{~dB}(10 \mathrm{dBi})$, where the beam response probably becames highly difficult to measure inflight; $\theta_{\mathrm{bf}, 2} \sim 5^{\circ}$ roughly divides pattern regions where significant response variations occur on angular scales less than $1^{\circ}$ from those where they occur on $\sim$ degree or much larger scales.

The observed antenna temperature is given by

$T_{\mathrm{a}, \mathrm{obs}}=\frac{\int J\left(\theta_{\mathrm{bf}}, \phi_{\mathrm{bf}}\right) T_{\mathrm{a}}\left(\theta_{\mathrm{bf}}, \phi_{\mathrm{bf}}\right) \mathrm{d} \Omega}{\int J\left(\theta_{\mathrm{bf}}, \phi_{\mathrm{bf}}\right) \mathrm{d} \Omega}$,

where $J$ and $T_{\mathrm{a}}$ are the antenna response and the sky antenna temperature in the direction given by $\theta_{\mathrm{bf}}, \phi_{\mathrm{bf}}$.

The convolution of antenna response with the sky and the integration of the antenna pattern is simply computed by adding the contributions from all the pixels within the considered solid angle, at resolutions corresponding to $n_{\text {side }}=1024,512,64$ respectively for the main, intermediate and far pattern, in order to take accurately into account the pattern response variations. The main pattern is given in equispaced cartesian coordinates with $\Delta x=\Delta y=0.0005$. The intermediate pattern and the far pattern are provided in equispaced "GRASP8" polar grids with $\Delta \phi_{\mathrm{bf}}^{\prime}=10^{\circ}$ and $\Delta \theta_{\mathrm{bf}}^{\prime}=0.1^{\circ}$ and $0.5^{\circ}$, respectively. When we extract all the pixels in the sky that contribute to the convolution within the considered solid angle, the exact central position of each pixel typically does not coincide with a grid point where the pattern is known. Simple standard bilinear interpolation (Press et al. 1992) on the pattern grid has been implemented: this is fast, robust and accurate enough for the present purposes.

An estimate of the error introduced by the above discretizations and interpolation/computation methods can be provided by comparing the convolutions obtained with different values of $n_{\text {side }}$, for example by increasing $n_{\text {side }}$ to 1024 for the intermediate pattern convolution. The numerical error is negligible $(\lesssim 1 \mu \mathrm{K}, \lesssim 0.5 \mu \mathrm{K}$ or $\lesssim 0.2 \mu \mathrm{K}$ for the convolutions with the main, intermediate and far pattern, respectively). Thus, the real uncertainty of the currently available straylight analyses is dominated by the uncertainty of our knowledge of the antenna pattern (particularly for the intermediate pattern, see Sect. 2.2) and of the Galaxy emission.

\subsection{Tests with schematic skies and patterns}

Checking the consistency of the part of the code that computes the signal entering in the main beam and in the intermediate pattern is quite direct: we simply expect that the maps extracted from the corresponding data streams are respectively very similar or roughly proportional (according to the fractional signal entering at $\left.1.2^{\circ} \leq \theta_{\mathrm{bf}} \leq 5^{\circ}\right)$ to Galactic emission pattern, except for the beam smoothing.

Testing the validity of the computation of the signal entering the far pattern is not immediate, because it does not reflect in a simple way the Galactic emission pattern.
We have verified the consistency of our simulation code by exploring simple cases for which we can easily predict the large-scale symmetries of the maps derived from the data streams observed by the far pattern. We have assumed a simple antenna pattern, centred on the optical axis and perfectly symmetric in $\phi_{\mathrm{bf}}$, given by the sum of two Gaussian shapes, one for the main beam and one for the main spillover located at $90^{\circ}$ from the main beam centre, plus a constant low response level. We have performed tests with the following different very simple input skies: i) a spot at North Galactic pole: it produces a map with a well defined slab on the Galactic plane; ii) a slab on the ecliptic plane: the corresponding map shows a signal maximum at the ecliptic poles and decreasing toward the ecliptic plane; iii) a slab on the Galactic plane: the corresponding map shows a signal maximum at the Galactic poles and decreasing toward the Galactic plane, where it exhibits small longitudinal modulations related to those of the solid angle subtended by the main spillover (this is due to the scanning symmetry with respect to ecliptic coordinates and not with respect to Galactic ones). We have also verified that the angular power spectrum of the these maps presents a main peak at the multipole $\ell=2$ and secondary peaks at its harmonic frequencies, as expected from the $90^{\circ}$ symmetry of the adopted far pattern.

\section{Simulations results}

We have considered two options for the PLANCK Carrier configuration. The first is exactly that considered by de Maagt et al. (1998) with $\alpha=80^{\circ}$ and including shields. In the second case we have used the same optical results but with $\alpha=90^{\circ}$ : the corresponding results are then only indicative, being not perfectly consistent because the spacecraft design would be slightly different for this configuration; on the other hand, this case is instructive because it allows us to start addressing the question of the dependence of GSC on a basic parameter of the scanning strategy. We have considered the antenna pattern at $30 \mathrm{GHz}$ with the beam centre located at $\theta_{\mathrm{B}}=5.62^{\circ}$ and $\phi_{\mathrm{B}}=126.03^{\circ}$.

\subsection{Analysis of the scan circle data streams}

Figures 4 and 5 show the absolute signals entering the main, intermediate and far pattern and their ratios for the data streams of three representative scan circles for $\alpha=80^{\circ}$ and $90^{\circ}$, respectively.

Note that the signal entering the intermediate pattern is roughly proportional to that in the main beam: two main relative maxima typically appear, related to the two crossings of the Galactic plane. The signal from the far sidelobes exhibits a clearly different and shifted angular behaviour, although two main relative maxima are again typically present. These are mainly due to the contributions from the pattern features (1a) in the cases of the left and central panels, and to the main spillover (2a) in the case of the right panels, as they cross the Galactic 

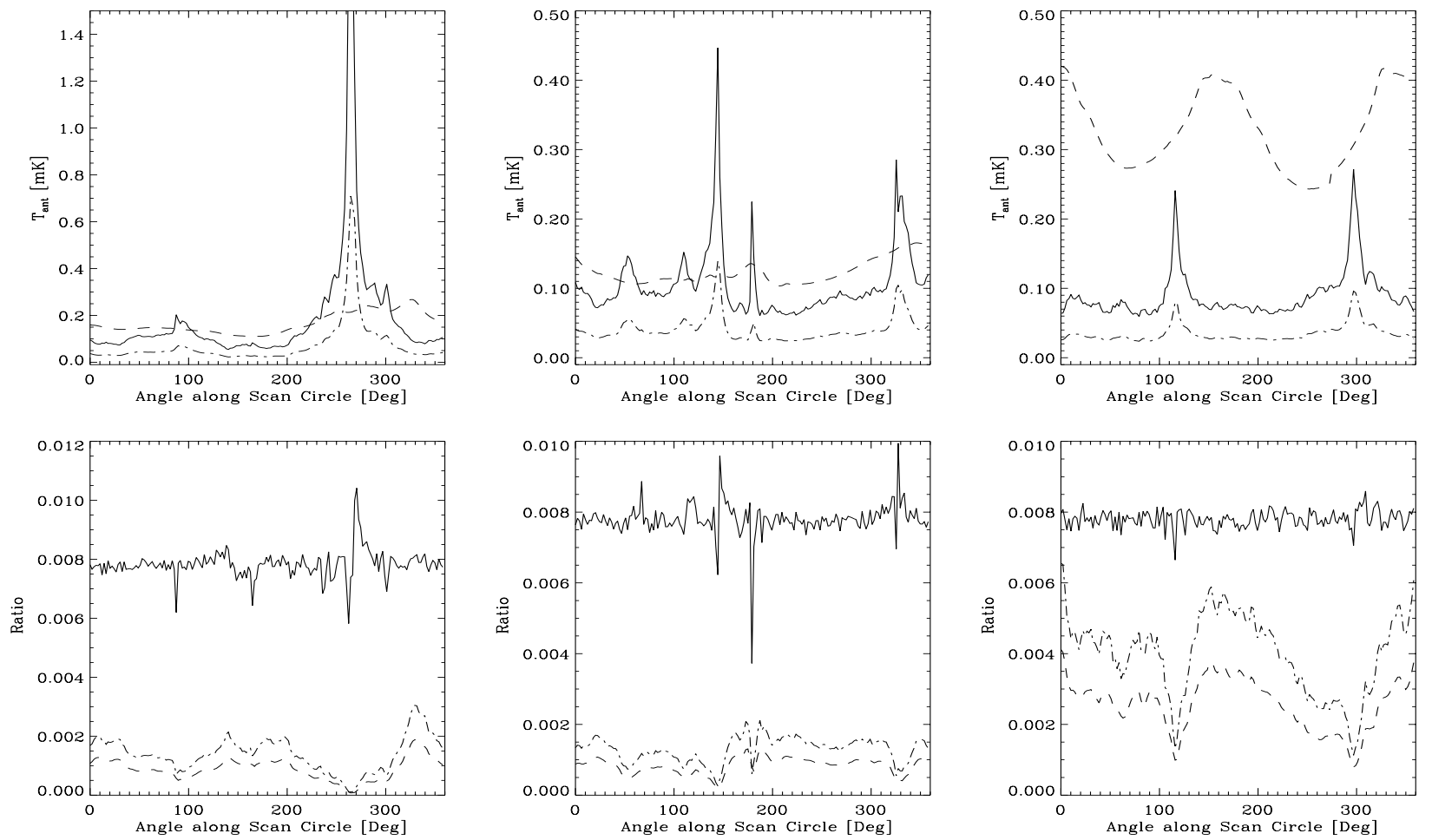

Fig. 4. Top panels: absolute signals entering the main (solid lines), intermediate (multiplied by 50 for graphic purposes, dotteddashed lines) and far (multiplied by 100, dashed lines) pattern for the case $\alpha=80^{\circ}$ for three scan circles corresponding to spin axis longitudes of $0^{\circ}, 120^{\circ}$ and $270^{\circ}$ from left to right. The angle along the scan circle, ranging between $0^{\circ}$ and $360^{\circ}$, is set to 0 at the beginning of the stream from each scan circle. Bottom panels: the ratios between the signals shown in the top panels, intermediate/main (solid lines), far/main (multiplied by 0.1 , dotted-dashed line) and far/intermediate (multiplied by $5 \times 10^{-4}$, dashed lines).

plane. Note in fact the displacement between the maximum signal from the main and far pattern, of few tens of degrees (several tens of degrees) for the left and central panel (right panel).

We have applied the Fourier series decomposition (see Fig. 6) described by Eq. (1) to the sum of intermediate and far pattern (i.e. for $\theta_{\mathrm{bf}} \geq 1.2^{\circ}$ ) data streams from the scan circles shown in Figs. 4 and 5 .

The same decomposition has been applied to white noise data streams, computed according to the LFI sensitivity at $30 \mathrm{GHz}$ (see, e.g., Maino et al. 1999) averaged over a number of scan circles that spans an ecliptic longitude length arc equal to the $F W H M=33^{\prime}$, i.e. essentially the sensitivity corresponding to a half-year mission. The white noise power is above that of the signal entering at $\theta_{\mathrm{bf}} \geq 1.2^{\circ}$, for practically all the modes $m \gtrsim 3$, becaming $\approx 10$ times larger at $m \approx 10$; this is essentially due to the strong decreasing of Galaxy fluctuations at small angular scales.

No significant differences are found by varying $\alpha$ from $80^{\circ}$ to $90^{\circ}$; for this reason and for the sake of simplicity, we will show in what follows only the results for $\alpha=$ $80^{\circ}$, the angle for which the optical simulations have been appropriately performed.

All simulated data streams for a 1 yr mission are shown in Fig. 7 for the case $\alpha=80^{\circ}$ (similar patterns are obtained in the case $\alpha=90^{\circ}$ ). In the right panel, note the vertical high signal line at $\lambda \simeq 270^{\circ}$, corresponding to the main spillover (2a), and the two high signal features, close to this line, at about $(\lambda, \beta) \sim\left(180^{\circ}, 0^{\circ}\right)$ and $(\lambda, \beta) \sim\left(360^{\circ},-160^{\circ}\right)$, corresponding to the pattern features at few tens of degrees from the beam centre. Note also how the azimuthally asymmetric far pattern reflects in the large difference between the two halfs (along $\lambda$ axis) of the right panel of Fig. 7 corresponding to the first and second six months of observation. This redundancy can be exploited for an efficient subtraction of GSC in the data analysis.

\subsection{Straylight contamination maps and angular power spectrum}

By coadding the data streams as described in Sect. 2.5 we can obtain the corresponding maps. This is shown in Fig. 8 for the case $\alpha=80^{\circ}$, by coadding the simulated data from the whole year.

As apparent in Fig. 8, the map from the intermediate pattern is roughly proportional to that derived from the main pattern; the relative intensities are roughly scaled by the fraction of integrated response entering the two portions of the antenna pattern.

On the contrary, the sky "observed" by the far pattern is very different. The signal is higher close to the Galactic plane, because of the features in the antenna 

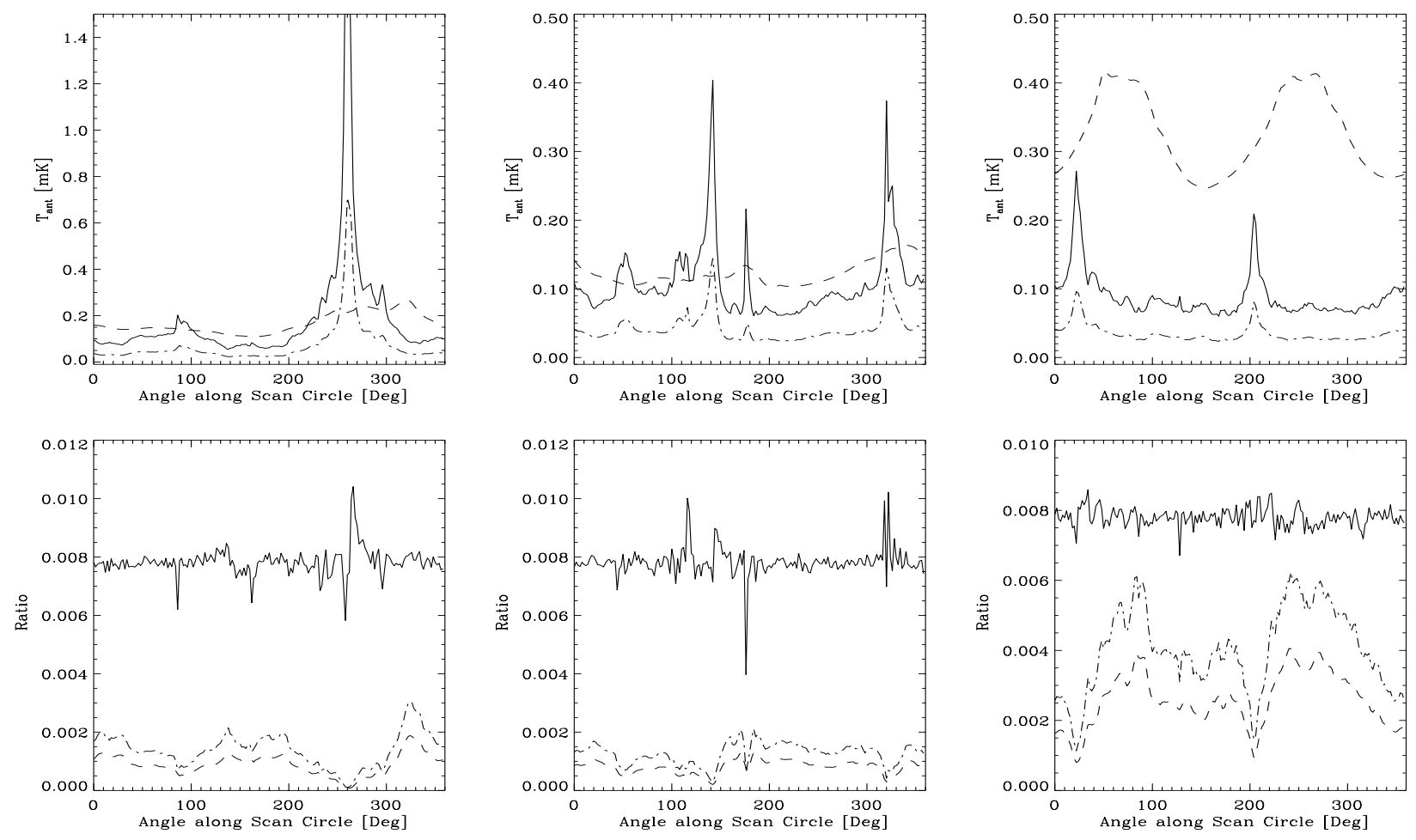

Fig. 5. The same as in previous figure, but for the case $\alpha=90^{\circ}$.

pattern within $10^{\circ}-20^{\circ}$ from the main beam, and at about $90^{\circ}$ from the Galactic plane, because of the signal entering the main spillover $(2 \mathrm{a})$.

We have computed the angular power spectra of these GSC maps (see Fig. 9) and compared them with the theoretical angular power spectrum of the white noise for a single $30 \mathrm{GHz}$ receiver and for four receivers and with a typical CMB anisotropy angular power spectrum (a tilted $-n_{\mathrm{p}}=0.9$ - power spectrum with standard CDM cosmological parameters and approximately COBE normalized) and with that of Galaxy fluctuations as seen by the main pattern. The most important contamination in terms of angular power spectrum derives from the signal entering in the intermediate pattern when all the sky is considered; on the contrary, if we consider only the regions at $|b| \geq 30^{\circ}$, more crucial for PLANCK main science, the largest contributions to the GSC power spectrum derive from the far sidelobes. In general, the GSC power spectrum is larger than the white noise one at low multipoles $(\ell \lesssim 5)$ but their ratio becames less than $\simeq 1 / 10$ at $\ell \simeq 50$ and decreases further at larger multipoles, due to their different dependence on $\ell^{4}$.

${ }^{4}$ The increase of the power of GSC from far sidelobes at $\ell \gtrsim 50$, evident in the plot, is not an intrinsic effect of the GSC but is generated by the "stripe" corresponding to the sky region observed a single time (see also Fig. 8) in the current $1 \mathrm{yr}$ simulation with $\alpha=80^{\circ}$ and off-axis beam.

\section{Comparison with other sources of noise}

Many other sources of contamination, both instrumental and astrophysical in origin, may affect PLANCK observations. We approach here a first comparison among the effects introduced by some of these systematics.

\subsection{Other kinds of instrumental noise}

The impact of main beam distortions introduced by optical aberrations on PLANCK measurements has been carefully studied in several works (see, e.g., Burigana et al. 1998, 2000a; Mandolesi et al. 1997, 2000a). Burigana et al. (1998) discussed the impact of the main beam distortions for the representative case of an elliptical main beam shape. In general, the absolute rms additional noise, in the range of few $\mu \mathrm{K}$, increases with the beam ellipticity. The combined effect of main beam distortions and of Galaxy emission fluctuations increases the additional error at $\sim 30 \mathrm{GHz}$ by a factor $\simeq 3$ with respect to the case of the essentially pure CMB fluctuation sky at high Galactic latitudes, whereas it produces only a small additional effect at higher LFI frequencies. In addition, the combined effect of main beam distortions and extragalactic source fluctuations is found to be very small at all LFI frequencies (Burigana et al. 2000a) compared to the noise induced by beam distortions in the case of a pure CMB sky. Then, we focus further on the impact of the main beam distortions on the determination of angular power spectrum of CMB fluctuations by considering the idealized case of the above pure CMB sky fluctuations. The kind and the magnitude of optical distortions depend on the details of the optical 

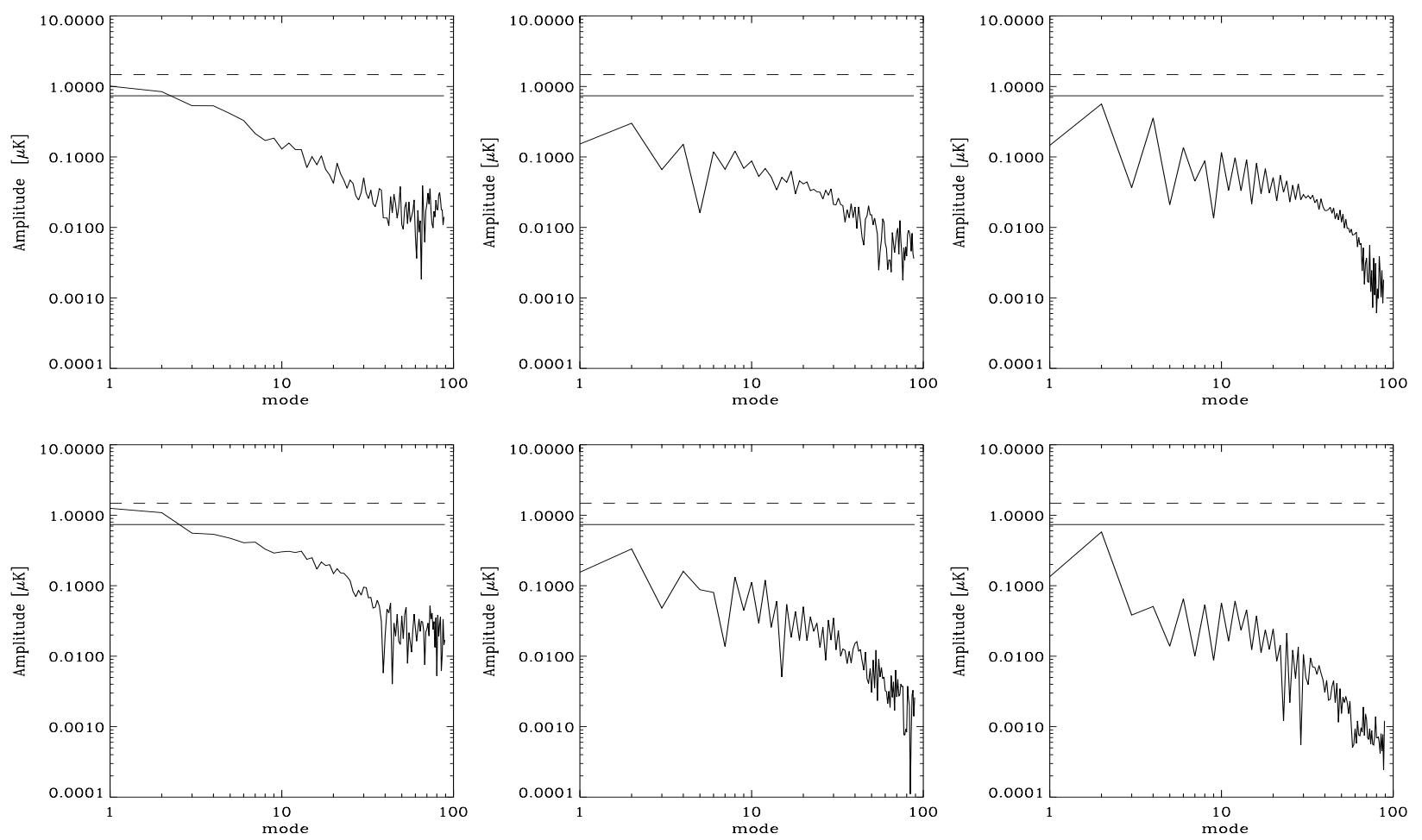

Fig. 6. Fourier decomposition, see Eq. (1), of the signal at $\theta_{\mathrm{bf}} \geq 1.2^{\circ}$ from the scan circles of Fig. $4\left(\alpha=80^{\circ}\right.$, top panels) and $5\left(\alpha=90^{\circ}\right.$, bottom panels). From the left to right: spin axis longitudes of $0^{\circ}, 120^{\circ}$ and $270^{\circ}$. Dashed and solid horizontal lines represent the white noise for one and four receivers, respectively.

design; for aplanatic configurations currently under study (see, e.g., Villa et al. 1998; Mandolesi et al. 2000b) the typical main beam shape is roughly elliptical owing to the strong reduction of the coma distortion. We computed a full year simplified simulation both for a pure symmetric Gaussian beam with $F W H M=\sigma \sqrt{8 \ln 2}=30^{\prime}$ and for an elliptical Gaussian beam with axial ratio $r=1.3$ and with the same effective resolution $\left(\sqrt{\sigma_{x} \sigma_{y}}=\sigma\right)$ of the symmetric beam $(r=1)$. We shift the spin axis at steps of $5^{\prime}$ and consider a step of $5^{\prime}$ between two samplings on the same scan circle. We computed the difference between the maps obtained from the elliptical and the symmetric beam by coadding the corresponding data streams and calculate the angular power spectrum of this difference map in order to understand which range of multipoles is mainly affected. As expected (see Fig. 10), this effect is particularly relevant at quite large multipoles, close to the CMB peak, where GSC power significantly decreases: the magnitude, of course, is related to the value of $r$.

From optical simulations we know that $r$ typically increases with the distance from the beam centre. From the present simulations we infer that a value of $r$ smaller than 1.3 , say less than $\simeq 1.1(\simeq 1.2)$ when the response level normalized to the maximum is less than $\simeq-3 \mathrm{~dB}(\simeq-20 \mathrm{~dB})$, is good enough to avoid significant contamination in the data, in agreement with the indications inferred on the basis of the approximations of Burigana et al. (1998) for the rms noise added by a main beam elliptical distortion.

The $1 / f$ noise due to amplifier noise temperature fluctuations induced by gain fluctuations in the PLANCK LFI receiver and its dependence on the relevant instrumental parameters has been studied by Seiffert et al. (1997). It introduces additional noise in PLANCK observations which appears as stripes in final maps (Janssen et al. 1996) owing to the particular PLANCK scanning strategy. We have recently carried out detailed studies (Maino et al. 1999 and reference therein) on its effect on PLANCK LFI measurements and on the efficiency of destriping algorithms based on the use of the crossings between different scan circles (Bersanelli et al. 1996; Delabrouille 1998) for a wide set of PlancK scanning strategies. We extend here their simulations by relaxing the hypothesis of a symmetric beam to study the impact of main beam distortion on the destriping algorithm. In Fig. 11 we show the angular power spectrum of the receiver noise before and after applying the destriping algorithm when we include also the above elliptical distortion, for a simple scanning strategy with $\alpha=90^{\circ}$ and a beam location at $\theta_{\mathrm{B}}=2.8^{\circ}, \phi_{\mathrm{B}}=45^{\circ}$, a "mean" choice regarding the destriping efficiency (Maino et al. 1999). We find that the destriping efficiency is not significantly affected by the additional uncertainty introduced by the "systematic" differences among the observed temperatures resulting from different orientations of the main beam at the crossing points of different scan circles.

As is evident by comparing Figs. 9-11, there is a crucial difference between the angular power spectra of GSC, main beam distortion induced noise and $1 / f$ noise. The GSC affects particularly the determination of the CMB angular power spectrum at low multipoles, whereas main beam distortions are critical at large multipoles. The $1 / f$ 

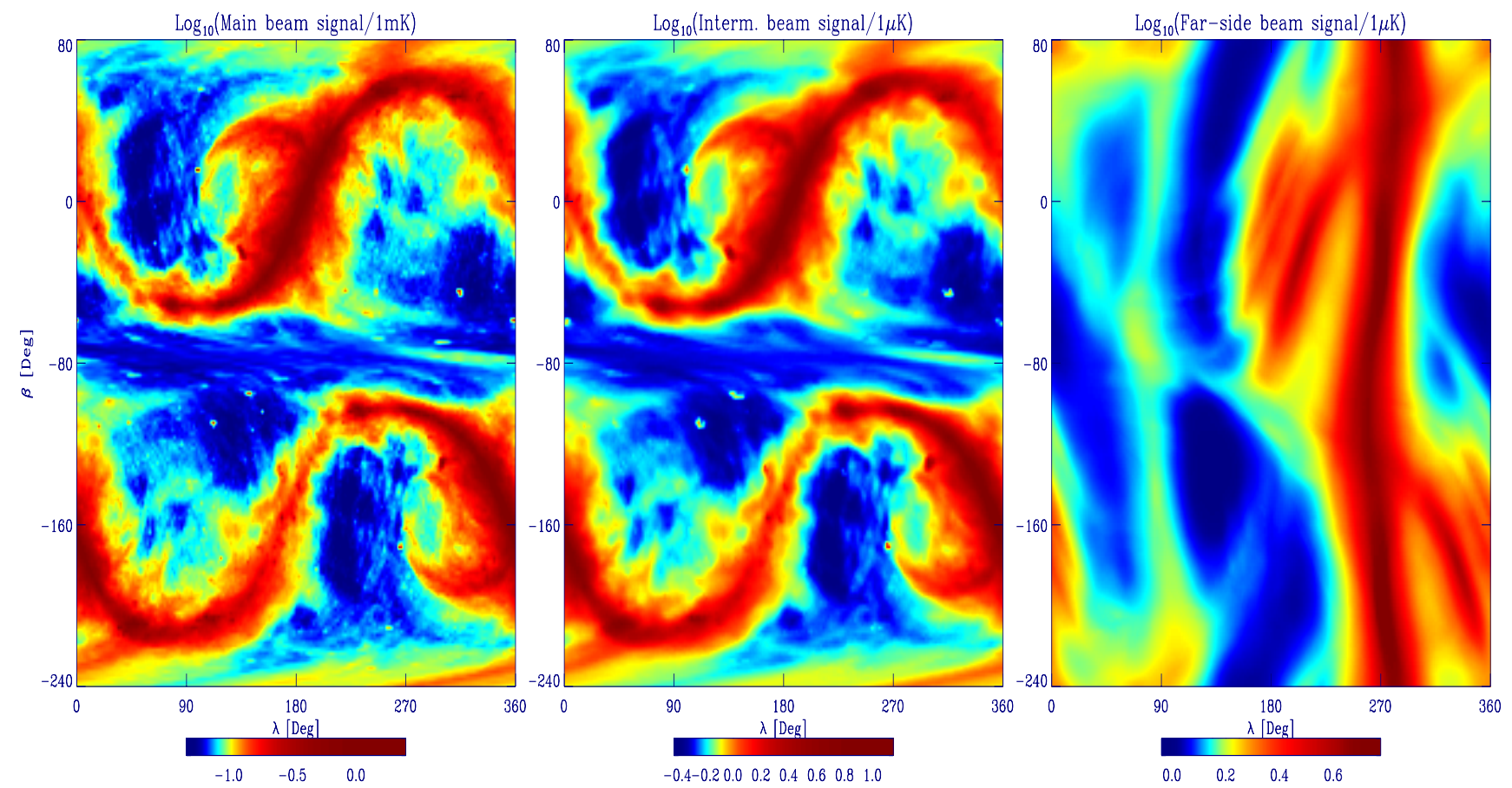

Fig. 7. Synthetic view of the data stream from all scan circles for $\alpha=80^{\circ}$. The ecliptic coordinates $\lambda$ and $\beta$ refer here to the longitude and latitude of the telescope axis. [For graphic purposes, in this plot the range of $\beta$ between $-80^{\circ}$ and $-240^{\circ}$ refer to the second half of each scan circle]. Compare with Fig. 4.

noise affects both high and low multipoles, but destriping algorithms are particularly efficient at removing high multipole features in the power spectrum. It is clear that all these effects have to be reduced both via hardware and software. The $1 / f$ noise can be reduced independently of the other two, its magnitude being related essentially to the instrument stability and to the scanning strategy (Maino et al. 1999). On the other hand, a compromise has to be reached between GSC and main beam distortion noise, both being related mainly to the optical design. As discussed in Mandolesi et al. (2000a), for a given telescope design, their relative weight is controlled by the edgetaper. The optical design has to be optimized to find a trade-off to reduce the combined impact of these two effects.

\subsection{Astrophysical contamination}

The impact of foreground on the primary cosmological goal of the PLANCK mission has been extensively studied in the literature for the areas concerning both Galactic and extragalactic contaminations, of discrete and diffuse origin; PLANCK itself will be a good opportunity for studying cluster physics, many classes of extragalactic and Galactic sources and the diffuse emission from the Galaxy (see, e.g., De Zotti 1999a and references therein). Many approaches have been studied to separate the different components of the microwave sky and for deriving their angular power spectra (see, e.g., Tegmark \& Esftathiou 1996; Hobson et al. 1998; Baccigalupi et al. 2000b, and references therein).
We consider here the foreground impact on CMB science and their comparison with the effect of instrumental systematics. In Fig. 12 we report several estimates of the angular power spectra of different astrophysical components.

Though depending on the considered region, the Galaxy angular power spectrum, expressed in terms of $\delta T_{\ell}$, is known to decrease with multipole order $\ell$ : we show here the power spectrum derived from the map observed by the adopted main pattern by cutting (lower thin dotted-dashed line, "CG") or not (upper thin dotteddashed line, "WG") the region at $|b| \leq 30^{\circ}$ and the power spectra proposed by Tegmark \& Esftathiou (1996) for freefree (thick dotted line, "FF"), synchrotron (upper thick dotted-dashed line, "S") and dust (upper thick dashed line, "D") emission at relevant Galactic latitudes. We also show for comparison the power spectra for synchrotron (lower thick dotted-dashed line, "s") and dust (lower thick dashed line, "d") as derived by Prunet et al.(1998) and Bouchet et al. (1999) for a sky patch at medium latitudes. Of course, Galaxy contamination strongly depends on the considered region.

In terms of $\delta T_{\ell}$, Poisson fluctuations from extragalactic unresolved discrete sources increase approximately proportionally to the multipole $\ell$. We show here, separately for radiosource (thin solid lines, "R") and the far infrared galaxy (thin dashed lines, "IR"), the Poisson fluctuation power spectra predicted by Toffolatti et al. (1998) as recently revised by Toffolatti et al. (1999), De Zotti et al. (1999b) and references therein, on the basis of current source counts and assuming evolution models and 

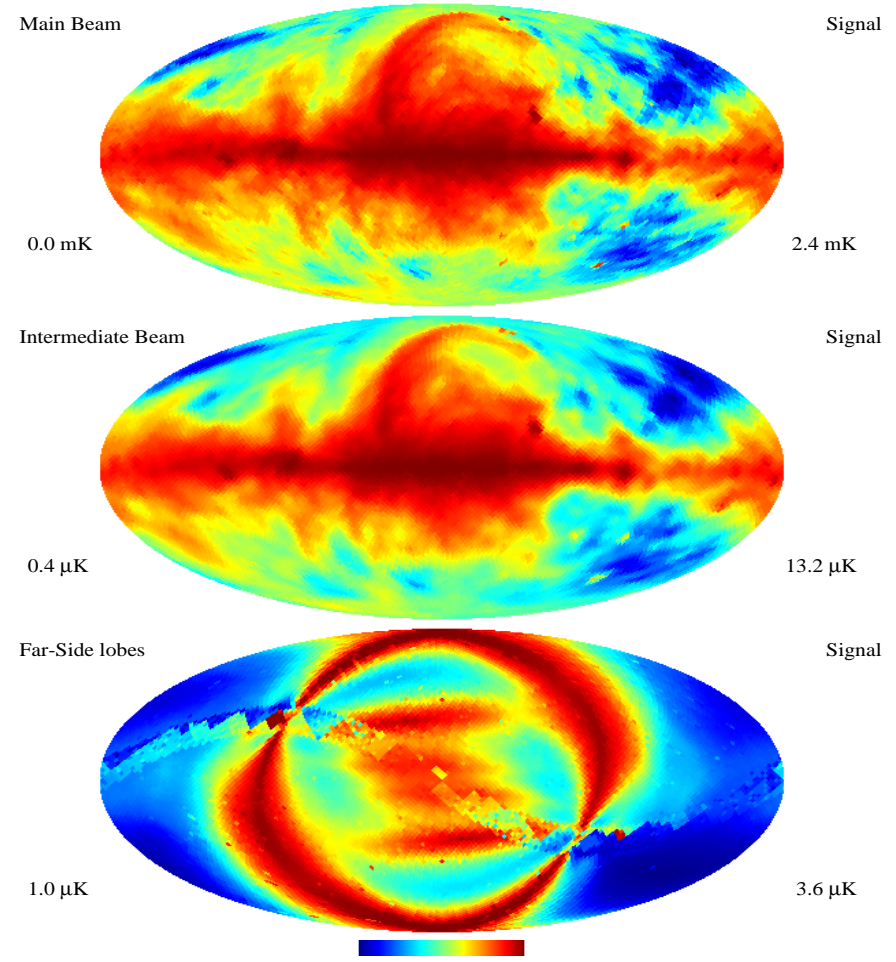

Fig. 8. Maps of GSC in Galactic coordinates from the coadding of the simulated data of the whole year for the case $\alpha=80^{\circ}$. [For graphic purposes, the pixels close to the ecliptic poles, not observed because of the adopted scanning strategy parameters, have been filled with values close to those of the adjacent pixels]. In the bottom panel, note the two arcs corresponding to the main spillover (2a) and the central contaminations corresponding to the pattern features at a few tens of degrees from the beam centre. They clearly correspond to the features in the right panel of Fig. 7. Note also the wide "stripe" connecting the two ecliptic poles, which corresponds to the sky region observed a single time in the current 1 yr simulation, owing to the considered effective scanning strategy. [The color at the left (right) edge of the color bar refers to the minimum (maximum) temperature reported in each panel].

spectra in agreement with current data, when sources above $1 \mathrm{Jy}$ (upper curves) or $100 \mathrm{mJy}$ (lower curves) are detected and subtracted. Of course radiosources dominate at low frequencies. We have taken into account here a Gaussian $\left(F W H M=33^{\prime}\right)$ beam smoothing in all cosmological and astrophysical angular power spectra and consequently neglected it in the receiver noise angular power spectrum.

At low multipoles, Galaxy contamination is larger then instrumental effects, dominated by the GSC (and possible residual $1 / f$ noise); on the other hand, if the Galaxy emission and anisotropy can be modelled at a few percent accuracy and at high Galactic latitudes, instrumental effects can became comparable with the residual Galaxy contamination.

If not appropriately taken into account in the data analysis, main beam distortions may introduce at high multipoles an additional contamination comparable with

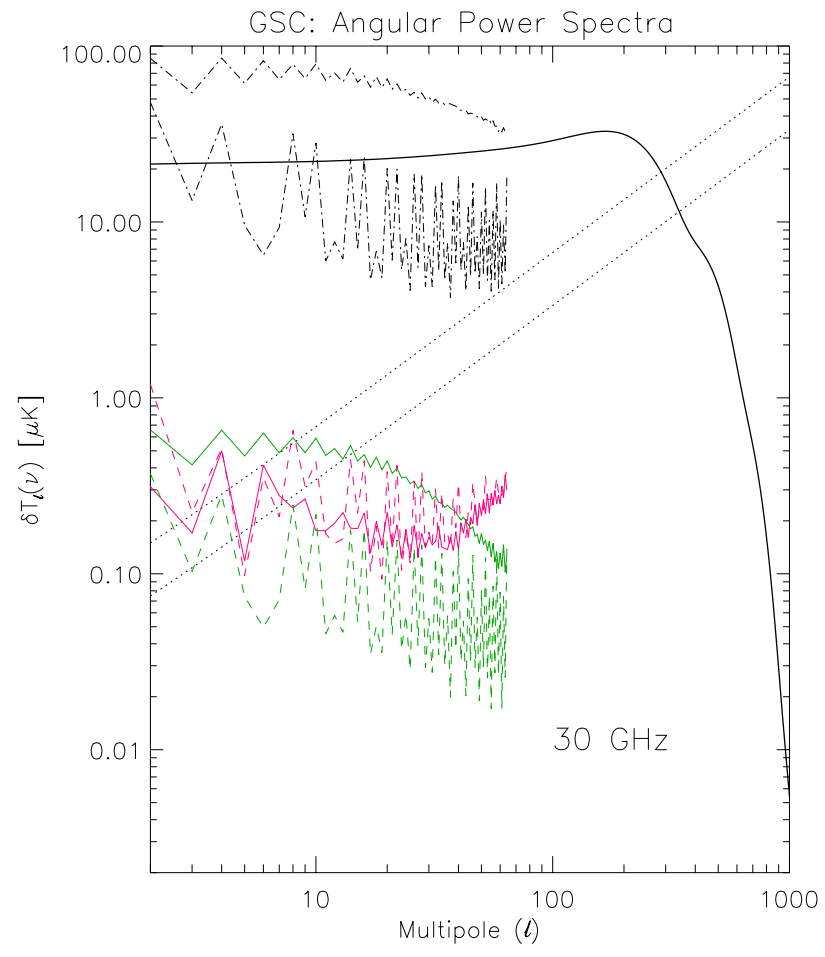

Fig. 9. Comparison between the angular power spectrum of GSC from different pattern regions and that of the CMB anisotropy (thick solid line) and of the receiver white noise (dotted lines; upper line: a single receiver; lower line: four receivers). Galaxy fluctuation power spectrum as seen by the main pattern without map cuts (upper dotted-dashed line) and by considering only the regions at $|b| \geq 30^{\circ}$ (lower dotteddashed line). Angular power spectra of GSC from the intermediate pattern without Galactic cut (upper solid - green - line) and at $|b| \geq 30^{\circ}$ (lower dashed - green - line) and from the far pattern without Galactic cut (lower solid - red - line) and at $|b| \geq 30^{\circ}$ (upper dashed - red - line). See also the text.

that introduced from radiosource fluctuations, after their subtraction at few hundreds mJy level.

\section{Conclusions}

We have studied the impact of GSC on PLANCK observations at $30 \mathrm{GHz}$, by considering different and complementary evaluation approaches: absolute and relative quantification of the impact on scan circle data streams, Fourier decomposition of scan circle signals, computation of maps of GSC and evaluation of their angular power spectra. These different methods allow us to focus on different aspects of GSC.

No relevant differences are found by varying the angle $\alpha$ between the spin axis and the telescope line of sight from $80^{\circ}$ to $90^{\circ}$.

Our simulations show that the GSC peaks at values of about $15 \mu \mathrm{K}$ (a value comparable with the sensitivity per pixel), mainly due to the signal entering at few degrees from the beam centre. Such values are found in the regions quite close to the Galactic plane, where in any case 


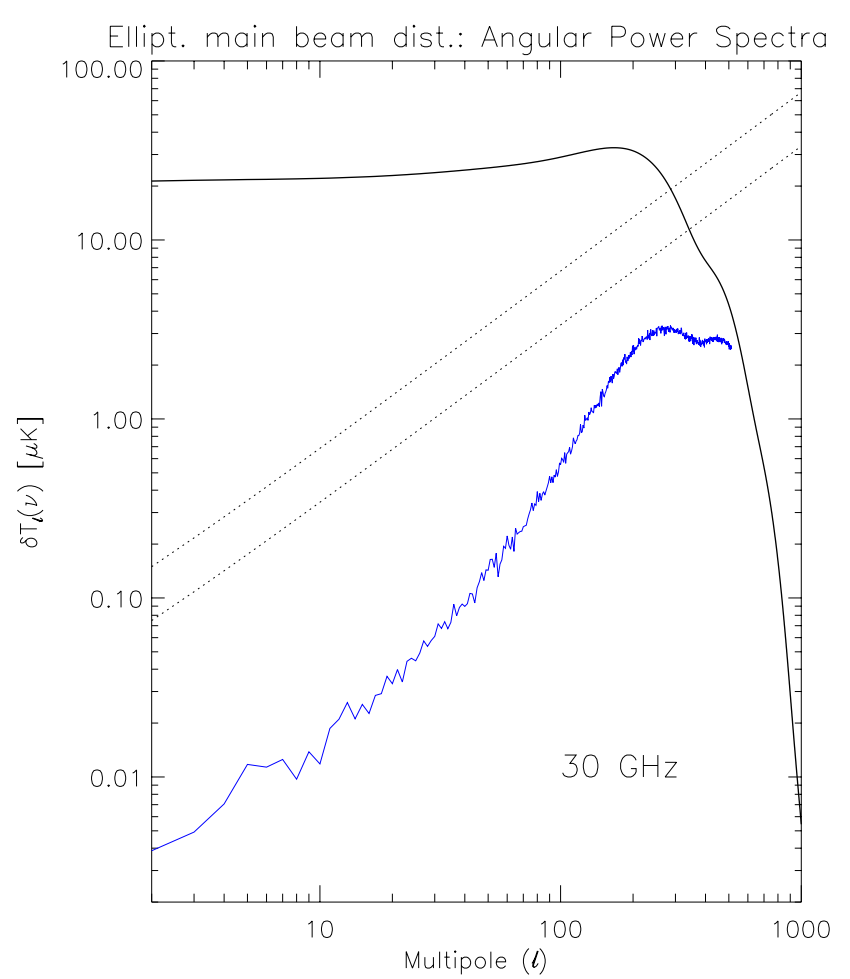

Fig. 10. Angular power spectrum introduced by an elliptical main beam distortion with $r=1.3$ (lower - blue - line). See also the text.

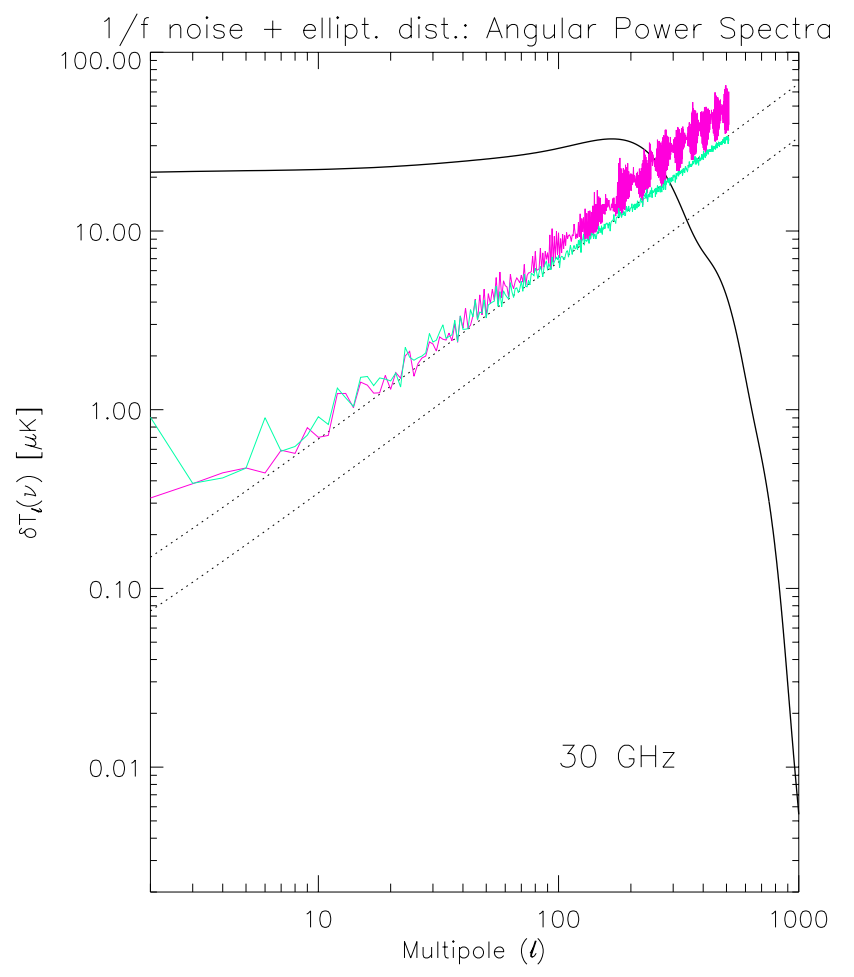

Fig. 11. Angular power spectrum of the $1 / f$ noise before (upper - red - line with blobs) and after (lower - green - line, close to the level of the theoretical white noise power spectrum for a single receiver) the destriping for the case of an elliptical main beam.

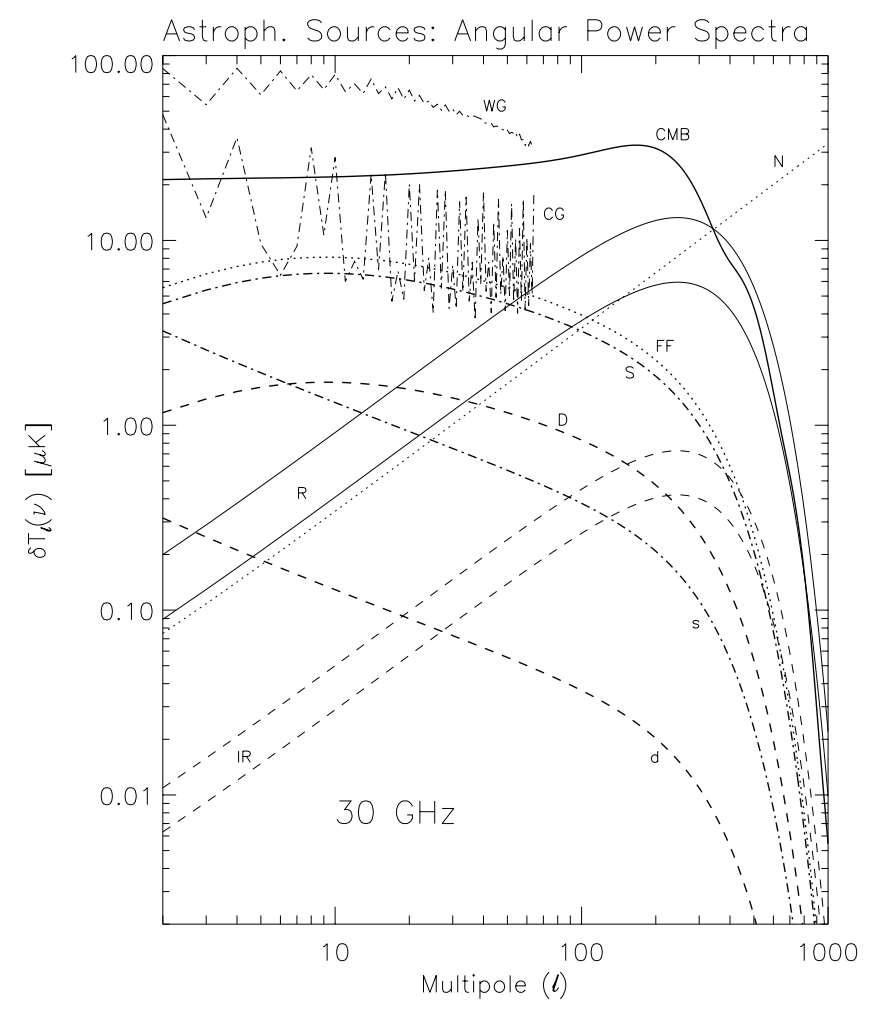

Fig. 12. Angular power spectrum of different sources of astrophysical contamination compared with the CMB angular power spectrum (thick solid line, labelled with "CMB") and that of the white noise power spectrum for four receivers (dotted line, "N"). See the text for the meaning of the other lines and labels.

the "direct" (i.e. observed by the main beam) contamination from the Galaxy prevents an accurate knowledge of CMB fluctuations, as it is next to impossible to remove the Galactic signals to accuracy better than $\simeq$ few $\%$. These large contamination values, although critical for CMB anisotropy measurements near the Galactic plane, are not crucial for the determination of Galaxy emission, which is several orders of magnitude larger.

By considering all the pixels in the sky, the typical values of GSC are less than $50 \%$ of the white noise sensitivity per pixel.

The most crucial contamination derives from the signal entering in the far pattern, in spite of its peak values, of about $4 \mu \mathrm{K}$, smaller than those obtained for the intermediate pattern regions. In fact, although this effect does not seem to be very large in amplitude (indeed, being nominally subdominant to the power spectrum of the expected receiver noise) it does dominate the GSC at medium and high Galactic latitudes, which are critical regions for the extraction of the best quality results on $\mathrm{CMB}$ anisotropy. This also could be critical for the PLANCK polarization measurements which will take advantage of the two patches close to the ecliptic poles where the sensitivity will be several times better than the average, according to the scanning strategy and the feed array arrangement. 
As expected on the basis of the behaviour of Galaxy emission angular power spectrum, the GSC affects the determination of the CMB angular power spectrum mainly in the low multipole region and much less at large multipoles, particularly when compared with the other instrumental effects considered here, the main beam distortion and the $1 / f$ noise. The additional noise introduced by the main beam distortion can be in principle subtracted in the data analysis, provided that the beam pattern is accurately reconstructed.

Of course, a substantial improvement in the data analysis is necessary to jointly treat all the systematics, of instrumental and astrophysical origin. From the telescope design point of view, the best optimization of the edge taper requires a trade-off between the main beam distortion and the GSC effects.

Color figures can be obtained from the electronic form of this paper or via e-mail request to

burigana@tesre.bo.cnr.it.

Acknowledgements. We acknowledge stimulating and helpful discussion with J. Delabrouille and J. L. Puget; we gratefully thank P. de Maagt and J. Tauber for having promptly provided us with their optical simulation results. We wish to thank the referee for constructive comments.

\section{References}

Baccigalupi, C., Burigana, C., Perrotta, F., et al. 2000a, A\&A, in press [astro-ph/0009135]

Baccigalupi, C., Bedini, L., Burigana, C., et al. 2000b, MNRAS, 318, 769

Balbi, A., Ade, P., Bock, J., et al. 2000, ApJ, 545, L1

Bennett, C. L., Banday, A. J., Gòrski, K. M., et al. 1996a, ApJ, 464, L1

Bennett, C. L., et al. 1996b, Amer. Astron. Soc. Meet., 88.05

Bersanelli, M., et al. 1996, ESA, COBRAS/SAMBA Report on the Phase A Study, D/SCI(96)3

Bouchet, F. R., Prunet, S., \& Sethi, S. K. 1999, MNRAS, 302, 663

Burigana, C., Malaspina, M., Mandolesi, N., et al. 1997, Int. Rep. TeSRE/CNR 198/1997, November [astro-ph/9906360]

Burigana, C., Maino, D., Mandolesi, N., et al. 1998, A\&AS, 130,551

Burigana, C., Maino, D., Mandolesi, N., et al. 2000a, Astroph. Lett. Comm., 37, 253

Burigana, C., Natoli, P., Vittorio, N., Mandolesi, N., \& Bersanelli, M. 2000b [astro-ph/0012273]

De Bernardis, P., Ade, P. A. R., Bock, J. J., et al. 2000, Nature, 404,955

Delabrouille, J. 1998, A\&AS, 127, 555

De Maagt, P., Polegre, A. M., \& Crone, G. 1998, Planck - Straylight Evaluation of the Carrier Configuration, Technical Report ESA, PT-TN-05967, 1/0

De Zotti, G., Toffolatti, L., Argüeso Gómez, F., et al. 1999a, Proceedings of the EC-TMR Conference $3 \mathrm{~K}$ Cosmology,
Roma, Italy, 5-10 October 1998, AIP Conference Proc. 476, ed. Maiani L., Melchiorri F., \& Vittorio N., 204 [astro-ph/9902103]

De Zotti, G., Gruppioni, C., Ciliegi, P., Burigana, C., \& Danese, L. 1999b, New Astron., 4, 481

Górski, K. M., Banday, A. J., Bennett, C. L., et al. 1996, ApJ, 464, L11

Górski, K. M., Hivon, E., \& Wandelt, B. D. 1998, Proceedings of the MPA/ESO Conference on Evolution of LargeScale Structure: from Recombination to Garching, ed. A. J. Banday, R. K. Sheth, \& L. Da Costa, 37 [astro-ph/9812350]

Hanany, S., Ade, P., Balbi, A., et al. 2000, ApJ, 545, L5

Hobson, M. P., Jones, A. W., Lasenby, A. N., \& Bouchet, F. R. 1998, MNRAS, 300, 1

Jaffe, A. H., Ade, P. A. R., Balbi, A., et al. 2000, Phys. Rev. Lett., submitted [astro-ph/0007333]

Janssen, M., Scott, D., White, M., et al. 1996 [astro-ph/9602009]

Lange, A. E., Ade, P. A. R., Bock, J. J., et al. 2000, Phys. Rev. D, submitted [astro-ph/0005004]

Maino, D., Burigana, C., Maltoni, M., et al. 1999, A\&AS, 140, 383

Mandolesi, N., Bersanelli, M., Burigana, C., et al. 1997, Int. Rep. TeSRE/CNR 199/1997, November

Mandolesi, N., et al. 1998, Planck LFI, A Proposal Submitted to the ESA

Mandolesi, N., Bersanelli, M., Burigana, C., et al. 2000a, A\&AS, 145, 323

Mandolesi, N., Bersanelli, M., Burigana, C., \& Villa, F. 2000b, Astroph. Lett. Comm., 37, 151

Muciaccia, P. F., Natoli, P., \& Vittorio, N. 1997, ApJ, 488, L63

Prunet, S., Sethi, S. K., Bouchet, F. R., \& Miville-Deschenes, M. A. 1998, A\&A, 339, 187

Press, W. H., Teukolsky, S. A., Vetterling, W. T., \& Flannery, B. P. 1992, Numerical Recipes in Fortran, second edition (Cambridge University Press)

Puget, J. L., et al. 1998, HFI for the Planck Mission, A Proposal Submitted to the ESA

Puget, J. L., \& Delabrouille, J. 1999, HFI Sidelobe Straylight Requirement Document - 2nd Revision - March 16, 1999

Seiffert, M., Burigana, C., Mandolesi, N., et al. 1997, Rev. Scient. Instrum., submitted

Smoot, G. F., Bennett, C. L., Kogut, A., et al. 1992, ApJ, 396, L1

Tegmark, M., \& Efstathiou, G. 1996, MNRAS, 281, 1297

Toffolatti, L., Argüeso Gómez, F., De Zotti, G., et al. 1998, MNRAS, 297, 117

Toffolatti, L., De Zotti, G., Argüeso Gómez, F., \& Burigana, C. 1999, in Proc. Intern. Conf.: Microwave Foregrounds, ed. A. de Oliveira-Costa, \& M. Tegmark, Conf. Ser., 181, 153 [astro-ph/9902343]

Villa, F., Mandolesi, N., \& Burigana, C. 1998, Int. Rep. TeSRE/CNR 221/1998, September

Wandelt, B. D., \& Górski, K. M. 2000, Phys. Rev. D, submitted [astro-ph/0008227], see also TAC report Planck Straylight Modelling, http://www.tac.dk/wandelt/papers.html 